\title{
Error Analysis of SDSS/LAMOST Stellar Radial Velocity Measurement
}

\author{
Fengfei Wang, Ali Luo and Haotong Zhang \\ National Astronomical Observatories, Chinese Academy of Sciences 20A Datun Road, \\ Chaoyang District, Beijing, China \\ email: ffwang@nao.cas.cn
}

\begin{abstract}
We select super high quality spectra of spectral type A, F, G, K, M stars from SDSS C DR7. We mix our spectra with noise and then measure their radial velocity. The analysis shows that the accuracy of K, M type stars is much better than A, F stars. With our relationship between the signal-to-noise and accuracy of radial velocity measurement of different spectral type, we provide a good reference for stellar study related to radial velocity.
\end{abstract}

Keywords. methods: statistical, techniques: radial velocities
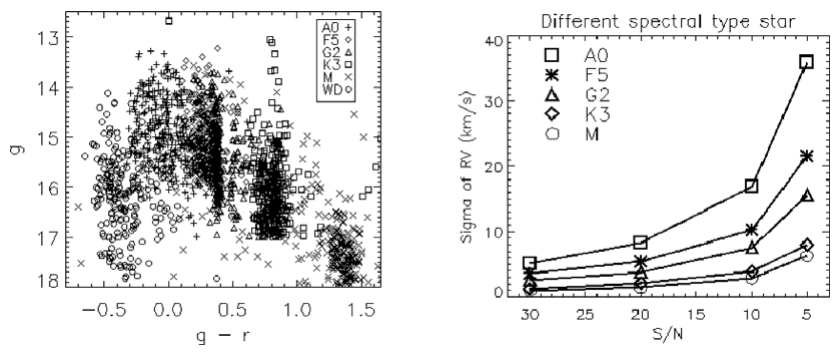

Figure 1. Left: The color-magnitude diagram of all different type spectra sample. Right: The accuracy of radial velocity of different spectral type stars at different $\mathrm{S} / \mathrm{N}$.

Large scale survey, such as SDSS(Stoughton et al. 2002) and LAMOST(Cui et al. 2012) generate millions of stellar spectra with their radial velocities measured by a templatematching algorithm utilizing ELODIE stellar spectra library(Moultaka et al. 2004). We select the best quality spectra from SDSS which are good enough to be considered as pure signal without much noise. The color-magnitude diagram of our sample is plotted in the left part of Fig. 1. We generate the noise-mixed spectra with $\mathrm{S} / \mathrm{N}=30,20,10$, and 5 , respectively, and measure the radial velocities of these synthetic spectra and compare with the original high quality spectra. The 1-sigma standard error of the radial velocity scatter is effected by the $\mathrm{S} / \mathrm{N}$. We summarize the results in the right part of Fig. 1 . The radial velocity of white dwarf stars shows an unacceptable error even for the high $\mathrm{S} / \mathrm{N}$ stellar spectra by our analysis. The radial velocity accuracy of different spectral type stars are discrepant. The early type stars, which have wide lines, are more sensitive to the noise and more difficult to gain precise radial velocities for than the late type stars.

\section{References}

Stoughton, C., Lupton, R., Bernardi, M., et al. 2002, AJ, 123, 485

Cui, X., Zhao, Y., Chu, Y., et al. 2012, Research in Astronomy and Astrophysics, 12, 1197

Moultaka, J., Ilovaisky, S., Prugniel, P., et al. 2004, PASP, 116, 693 